\title{
Propagation of squeezed radiation through amplifying or absorbing random media
}

\author{
M Patra and C W J Beenakker \\ Instituut Lorentz, Untversiteut Lelden, PO Box 9506, 2300 RA Leiden, The Netherlands \\ (Received 25 October 1999, revised manuscript received 12 January 2000, published 10 May 2000)
}

\begin{abstract}
We analyze how nonclassical features of squeezed radiation (in particular, the sub-Poissonian noise) are degraded when it is transmitted through an amplifying or absorbing medium with randomly located scattering centra Both the cases of direct photodetection and of homodyne detection are considered Explicit results are obtaned for the dependence of the Fano factor (the ratio of the norse power and the mean current) on the degree of squeezing of the incident state, on the length and the mean fiee path of the medium, the temperature, and on the absorption or amplification rate
\end{abstract}

PACS number(s) $4250 \mathrm{Dv}, 4225 \mathrm{Bs}, 4225 \mathrm{Dd}, 4250 \mathrm{Ar}$

\section{INTRODUCTION}

Squeezed radiation is in a state in which one of the quadratures of the electric field fluctuates less than the other $[1,2]$ Such a nonclassical state is useful, because the fluctuations in the photon flux can be reduced below that of a Poisson process - at the expense of enhanced fluctuations in the phase Sub-Poissonian noise is a delicate feature of the radiation, it is easily destroyed by the interaction with an absorbing or amplifying medium [3] The noise fiom spontancous-emission events is responsible for the degradation of the squeezing

Because of the fundamental and practical importance, there exists a considerable liteiature on the propagation of squeezed and other nonclassical states of light thiough absorbing or amplifying media We cite some of the most iecent papers on this topic [4-10] The main simplification of these investigations is the iestriction to systems in which the scattering is one-dimensional, such as parallel dielectı1c layers Each propagating mode can then be treated separately flom any other mode It is the purpose of the present paper to remove this restiction by presenting a genetal theory for three-dimensional scattering, and to apply it to a medium with randomly located scattering centra

Our work builds on a previous paper [11], in which we considered the propagation of a coherent state through such a random medium Physically, the problem considered heie is different because a coherent state has Poisson noise, so that the specific nonclassical features of squeezed radiation do not arise in Ref [11] Technically, the difference is that a squeezed state, as most other nonclassical states, lacks a diagonal representation in terms of coherent states $[1,2]$ We cannot therefore duectly extend the theory of Ref [11] to the propagation of squeezed states The basic idea of our approach remains the same The photodetection statistics of the transmitted radiation is related to that of the incident radiation by means of the scattering matrix of the medium The method of random-matrix theory [12] is then used to evaluate the noise properties of the transmitted radiation, averaged over an ensemble of random media with different positions of the scatterers

The outline of this paper is as follows In Sec II we fitst summarize the scattering formalism and then show how the characteistic function of the state of the transmitted radia- tion can be obtained from that of the incident state This allows us to compute the photocount statistics as measured in dinect detection (Sec III) and in homodyne photodetection measurements (Sec IV) The expressions in Secs II-IV are geneially valid for any incident state In Sec $V$ we specialize to the case that the incident radiation is in an ideal squeezed state (also known as a squeezed state of minimal unceitainty, or as a two photon coherent state $[1,2]$ ) The statistics of direct and homodyne measuiements are expressed in teims of the degree of squeezing of the incident state The Fano factor, introduced in Sec VI, quantifies the degiee to which the squeezing has been destroyed by the propagation through an amplifying or absoibing medium The ensemble average of the Fano factor is then computed using random-matrix theory in Sec VII We conclude in Sec VIII

\section{SCATTERING FORMULATION}

We consider an amplifying or absorbing disordered medium embedded in a waveguide that supports $N(\omega)$ propagatıng modes at frequency $\omega$ The conceptual advantage of embedding the medium in a waveguide is that we can give a scattering foimulation in terms of a finite-dimensional matrix The outgoing radiation in mode $n$ is described by an annihilation operato $a_{n}^{\text {out }}(\omega)$, using the convention that modes $1,2, \quad, N$ are on the left-hand side of the medium and modes $N+1, \quad, 2 N$ are on the inght-hand side The vector $a^{\text {out }}$ consists of the operators $a_{1}^{\text {out }}, a_{2}^{\text {out }}, \quad, a_{2 N}^{\text {out }}$ Similarly, we define a vector $a^{\text {in }}$ for incoming radiation

These two sets of operators each satisfy the bosonic commutation relations

$$
\left[a_{n}(\omega), a_{m}^{\dagger}\left(\omega^{\prime}\right)\right]=\delta_{n m} \delta\left(\omega-\omega^{\prime}\right), \quad\left[a_{n}(\omega), a_{m}\left(\omega^{\prime}\right)\right]=0
$$

They are related by the input-output relations [13-15]

$$
\begin{aligned}
& a^{\text {out }}(\omega)=S(\omega) a^{\mathrm{nn}}(\omega)+Q(\omega) b(\omega), \\
& a^{\text {out }}(\omega)=S(\omega) a^{\mathrm{nn}}(\omega)+V(\omega) c^{\dagger}(\omega),
\end{aligned}
$$

whele the filst equation is for an absorbing medium and the second for an amplifying medium We have introduced the $2 N \times 2 N$ scattering matrix $S$, the $2 N \times 2 N$ matrices $Q$ and $V$, 
and the vectors $b$ and $c$ of $2 N$ bosonic operators The scattering matrix can be decomposed into four $N \times N$ reflection and transmission matrices,

$$
S=\left(\begin{array}{cc}
r^{\prime} & t^{\prime} \\
t & r
\end{array}\right)
$$

Recipiocity imposes the conditions $t^{\prime}=t^{T}, r=r^{T}$, and $r^{\prime}$ $=r^{\prime T}$

The operators $b$ and $c$ account for spontaneous emission in the medium They satisfy the bosonic commutation relations (2 1), hence

$$
Q Q^{\dagger}=I-S S^{\dagger}, \quad V V^{\dagger}=S S^{\dagger}-1
$$

The11 expectation values are

$$
\begin{gathered}
\left\langle b_{n}^{\dagger}(\omega) b_{m}\left(\omega^{\prime}\right)\right\rangle=\delta_{n m} \delta\left(\omega-\omega^{\prime}\right) f(\omega, T), \\
\left\langle c_{n}(\omega) c_{m}\left(\omega^{\prime}\right)\right\rangle=-\delta_{n m} \delta\left(\omega-\omega^{\prime}\right) f(\omega, T)
\end{gathered}
$$

The Bose-Einstem function

$$
f(\omega, T)=[\exp (\hbar \omega / k T)-1]^{-1}
$$

is evaluated at positive tempeiature $T$ for an absoibing medium and at negative temperature for an amplifying medium

It is convenient to discietize the frequency in infintesimally small steps of $\Delta$, so that $\omega_{p}=p \Delta$, and theat the frequency index $p$ as a separate vector index (1n addition to the mode index $n)$ Fo1 example, $a_{n p}^{\text {out }}=a_{n}^{\text {out }}\left(\omega_{p}\right)$ and $S_{n p n^{\prime} p^{\prime}}$ $=S_{n n^{\prime}}\left(\omega_{p}\right) \delta_{p p^{\prime}}$

The state of the outgoing radiation is described by the characteristic function

$$
\begin{aligned}
\chi_{\text {out }}(\eta)= & \left\langle\operatorname { e x p } \left(\Delta ^ { 1 / 2 } \sum _ { n p } \left[ a_{n}^{\text {out }}\left(\omega_{p}\right) \eta_{n}\left(\omega_{p}\right)\right.\right.\right. \\
& \left.\left.\left.-\eta_{n}^{*}\left(\omega_{p}\right) a_{n}^{\text {out }}\left(\omega_{p}\right)\right]\right)\right\rangle \\
= & \left\langle\exp \left[\Delta^{1 / 2}\left(a^{\text {out }} \eta-\eta^{\dagger} a^{\text {out }}\right)\right]\right\rangle,
\end{aligned}
$$

where $\langle\quad>$ indicates the expectation value of a normally ordered product of operators $a^{\text {out }}$ and $a^{\text {out }}$ (creation operators to the left of the anmihilation operatots) The vector $\eta$ has elements $\eta_{n p}=\eta_{n}\left(\omega_{p}\right)$ The density operator of the outgoing radiation is uniquely defined by the characteristic function $\chi_{\text {out }}[1]$ Similarly, the incoming state has a characteristic function,

$$
\chi_{\mathrm{in}}(\eta)=\left\langle\exp \left[\Delta^{1 / 2}\left(a^{1 \mathrm{n} \dagger} \eta-\eta^{\dagger} a^{\mathrm{in}}\right)\right]\right\rangle
$$

The characteristic function of the thermal radiation inside an absorbing medium is given by

$$
\begin{aligned}
\chi_{\mathrm{dbs}}(\eta) & =\left\langle\exp \left[\Delta^{1 / 2}\left(b^{\dagger} \eta-\eta^{\dagger} b\right)\right]\right\rangle \\
& =\exp \left(-\sum_{n p} \eta_{n p}^{*} f\left(\omega_{p}, T\right) \eta_{n p}\right) \\
& \equiv \exp \left(-\eta^{\dagger} f \eta\right)
\end{aligned}
$$

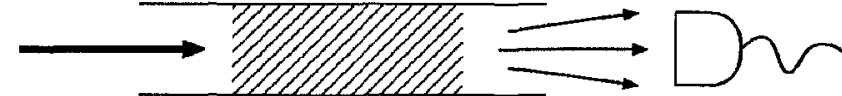

FIG 1 Schematic illustration of direct detection Radiation is incident on a random medium (shaded) The transmitted radiation is absorbed by a photodetector

In the final equality $f$ denotes the matrix with elements $f_{n p n^{\prime} p^{\prime}}=\delta_{n n^{\prime}} \delta_{p p^{\prime}} f\left(\omega_{p}, T\right)$ For an amplifying medium, replacing $b$ by $c^{\dagger}$ and normal ordering by antinormal ordering, one finds instead

$$
\chi_{\operatorname{mp}}(\eta)=\exp \left(\eta^{\dagger} f \eta\right)
$$

A combination of Eqs (2 2) and (2 4) with Eqs (2 7)(2 10) yields a relationship between the characteristic functions of the incoming and outgoing states,

$$
\chi_{\text {out }}(\eta)=\exp \left(-\eta^{\dagger}\left(1-S S^{\dagger}\right) f \eta\right) \chi_{\mathrm{In}}\left(S^{\dagger} \eta\right)
$$

This relation holds both for absoibing and amplifying media, because the difference in sign in the exponent of Eqs (29) and (2 10) is canceled by the difference in sign between $Q Q^{\dagger}=1-S S^{\dagger}$ and $V V^{\dagger}=-\left(1-S S^{\dagger}\right)$

\section{PHOTOCOUNT DISTRIBUTION}

The photocount distribution is the probability $P(n)$ that $n$ photons are absorbed by a photodetector within a certan time $\tau$ (see Fig 1) The factorial cumulants $\kappa_{j}$ of $P(n)$ [the fiust two being $\kappa_{1}=\bar{n}$ and $\kappa_{2}=\overline{n(n-1)}-\bar{n}^{2}$ ] are most easily obtained from the generating function [2]

$$
F(\xi)=\sum_{j=1}^{\infty} \frac{\kappa_{j} \xi^{J}}{j^{\prime}}=\ln \left(\sum_{n=0}^{\infty}(1+\xi)^{n} P(n)\right)
$$

The generating function is determined by a noimally ordered expectation value $[16,17]$,

$$
e^{F(\xi)}=\left\langle e^{\xi W}\right\rangle, \quad W=\int_{0}^{\tau} d t \sum_{n=1}^{2 N} d_{n} a_{n}^{\text {out } \dagger}(t) a_{n}^{\text {out }}(t)
$$

Here $d_{n} \in[0,1]$ is the detection efficiency of the $n$th mode and the time-dependent operators are defined as

$$
a_{n}^{\text {out }}(t)=(2 \pi)^{-1 / 2} \int_{0}^{\infty} d \omega e^{-t \omega t} a_{n}^{\text {out }}(\omega)
$$

Discretizing the frequencies as descitbed in Sec II, one can write

$$
W=\frac{\Delta^{2}}{2 \pi} \int_{0}^{\tau} d t \sum_{n} d_{n} \sum_{p p^{\prime}} e^{i \Delta\left(p-p^{\prime}\right) t} a_{n}^{\text {out } \dagger}\left(\omega_{p}\right) a_{n}^{\text {out }}\left(\omega_{p^{\prime}}\right)
$$

This expression can be simplified in the limit $\tau \rightarrow \infty$ of long counting tımes, when one can set $\Delta=2 \pi / \tau$ and use 


$$
\int_{0}^{\tau} e^{i \Delta\left(p-p^{\prime}\right) t} d t=\tau \delta_{p p^{\prime}}
$$

Hence, in the long-tıme limit the gener atıng function is given by

$$
\begin{aligned}
e^{\Gamma(\xi)} & =\left\langle\exp \left(\xi \Delta \sum_{n p} d_{n} a_{n}^{\text {out }}\left(\omega_{p}\right) a_{n}^{\text {out }}\left(\omega_{p}\right)\right)\right\rangle \\
& \equiv\left\langle\exp \left(\xi \Delta a^{\text {out } 1} \mathcal{D} a^{\text {out }}\right\rangle\right),
\end{aligned}
$$

where we have defined the matrix of detector efficiencres $\mathcal{D}_{n p n^{\prime} p^{\prime}}=d_{n} \delta_{n n^{\prime}} \delta_{p p^{\prime}}$

Comparing Eqs (27) and (36), we see that the generating function $F(\xi)$ can be obtaned from the characteristic function $\chi_{\text {out }}$ by convolution with a Gaussian,

$$
e^{F(\xi)}=\frac{1}{\operatorname{det}(-\xi \pi \mathcal{D})} \int d \eta \chi_{\text {out }}(\eta) \exp \left(\frac{1}{\xi} \eta^{\dagger} \mathcal{D}^{-1} \eta\right)
$$

where $\int d \eta$ is an mtegtation over the real and maginary parts of $\eta$ We now substitute the relation (211) between $\chi_{\text {out }}$ and $\chi_{1 \mathrm{n}}$, to anive at a telation between $F(\xi)$ and $\chi_{\mathrm{in}}$

$$
\begin{aligned}
e^{F(\xi)}= & \frac{1}{\operatorname{det}(-\xi \pi \mathcal{D})} \int d \eta \chi_{\mathrm{In}}\left(S^{\dagger} \eta\right) \\
& \times \exp \left(\frac{1}{\xi} \eta^{\dagger} \mathcal{D}^{-1} \eta-\eta^{\dagger}\left(1-S S^{\dagger}\right) f \eta\right)
\end{aligned}
$$

The fluctuations in the photocount ate partly due to thermal fluctuations, which would exist even without any incident radiation If we denote by $F_{\mathrm{th}}(\xi)$ the generating function of these thermal fluctuations, then $\mathrm{Eq}(38)$ can be wilten in the form

$$
\begin{gathered}
F(\xi)=F_{\mathrm{th}}(\xi)+\ln \left[\frac{1}{\operatorname{det}(\pi M)} \int d \eta \chi_{\mathrm{ln}}(\eta) \exp \left(-\eta^{\dagger} M^{-1} \eta\right)\right] \\
F_{\mathrm{th}}(\xi)=-\ln \operatorname{det}\left[1-\xi \mathcal{D}\left(\mathbb{1}-S S^{\dagger}\right) f\right]
\end{gathered}
$$

We have defined the Hermitian matrix

$$
M=-\xi S^{\dagger}\left[1-\xi \mathcal{D}\left(\mathbb{1}-S S^{\dagger}\right) f\right]^{-1} \mathcal{D} S,
$$

and we have performed a change of mtegiation variables from $\eta$ to $S^{\dagger} \eta$ [with Jacobian $\left.\operatorname{det}\left(S S^{\dagger}\right)\right]$

The expression (3 10) generalizes the result of Ref [15] to arbitrary detection-efficiency matrix $\mathcal{D}$ Returning to a continuous fiequency, it can be written as (recall that $\Delta$ $=2 \pi / \tau)$

$$
\begin{aligned}
F_{\text {th }}(\xi)= & -\frac{\tau}{2 \pi} \int_{0}^{\infty} d \omega \operatorname{In} \operatorname{det}\left(1-\xi D\left[1-S(\omega) S^{\dagger}(\omega)\right]\right. \\
& \times f(\omega, T))
\end{aligned}
$$

where $D$ is a $2 N \times 2 N$ diagonal matrix containing the detection efficiencies $d_{n}$ on the diagonal $\left(D_{n m}=d_{n} \delta_{n m}\right)$ The first two factorial cumulants are

$$
\begin{gathered}
\kappa_{1}^{\mathrm{th}}=\tau \int_{0}^{\infty} \frac{d \omega}{2 \pi} f(\omega, T) \operatorname{tr} D\left[1-S(\omega) S^{\dagger}(\omega)\right], \\
\kappa_{2}^{\mathrm{th}}=\tau \int_{0}^{\infty} \frac{d \omega}{2 \pi} f^{2}(\omega, T) \operatorname{tl}(D[1-S(\omega) S(\omega)])^{2}
\end{gathered}
$$

Note that all factorial cumulants depend linearly on the detection time $\tau$ in the long-time limit

If only the $N$ modes at one side of the waveguide are detected (with equal efficiency $d$ ), then $d_{n}=0$ for $1 \leqslant n \leqslant N$ and $d_{n}=d$ for $N+1 \leqslant n \leqslant 2 N$, hence

$$
\begin{aligned}
F_{\mathrm{t} 1}(\xi)= & -\frac{\tau}{2 \pi} \int_{0}^{\infty} d \omega \ln \operatorname{det}\left(1-\xi d\left[1-,(\omega),^{\dagger}(\omega)\right.\right. \\
& -t(\omega) t(\omega)] f(\omega, T))
\end{aligned}
$$

in agieement with Ref [18]

The difference $F(\xi)-F_{\mathrm{th}}(\xi)$ contains the noise from the incident radiation by itself as well as the excess noise due to beating of the incident radiation with the vacuum fluctuations If the incident radiation is in a coherent state, then $\chi_{1 \mathrm{n}}(\eta)=\exp \left(\alpha^{\dagger} \eta-\eta^{\dagger} \alpha\right)$ for some vector $\alpha$ (called the displacement vector) with elements $\alpha_{n p}=\alpha_{n}\left(\omega_{p}\right)$ Substitution into Eq (39) gives the generating function

$$
\begin{aligned}
F(\xi)= & F_{\mathrm{th}}(\xi)-\alpha^{\dagger} M \alpha \\
= & F_{\mathrm{th}}(\xi)+\frac{\tau \xi}{2 \pi} \int_{0}^{\infty} d \omega \alpha^{\dagger}(\omega) S^{\dagger}(\omega) \\
& \times\left\{\mathbb{1}-\xi D\left[\mathbb{1}-S(\omega) S^{\dagger}(\omega)\right] f(\omega, T)\right\}^{-1} \\
& \times D S(\omega) \alpha(\omega)
\end{aligned}
$$

The first two factorial cumulants are

$$
\begin{aligned}
\kappa_{1}=\tau \int_{0}^{\infty} \frac{d \omega}{2 \pi} \alpha^{\dagger}(\omega) S^{\dagger}(\omega) D S(\omega) \alpha(\omega)+\kappa_{1}^{\text {th }}, \\
\kappa_{2}=2 \tau \int_{0}^{\infty} \frac{d \omega}{2 \pi} f(\omega, T) \alpha^{\dagger}(\omega) S^{\dagger}(\omega) D\left[1-S(\omega) S^{\dagger}(\omega)\right] \\
\quad \times D S(\omega) \alpha(\omega)+\kappa_{2}^{\text {th }}
\end{aligned}
$$

If the incident coherent radiation is in a single mode $m_{0}$ and monochromatic with fiequency $\omega_{0}$, then Eqs (3 16)(3 18) simplify for detection in transmission to

$$
\begin{gathered}
F(\xi)=F_{\mathrm{th}}(\xi)+\tau \xi d I_{0}\left(t ^ { \dagger } \left\{\mathbb{1}-\xi d\left[\mathbb{1}-, r^{\dagger}-t t^{\dagger}\right]\right.\right. \\
\left.\left.\times f\left(\omega_{0}, T\right)\right\}^{-1} t\right)_{m_{0} m_{0}}, \\
\kappa_{1}=I_{0} \tau d\left[t^{\dagger} t\right]_{m_{0} m_{0}}+\kappa_{1}^{\mathrm{th}}, \\
\kappa_{1}^{\mathrm{th}}=\tau d \int_{0}^{\infty} \frac{d \omega}{2 \pi} f(\omega, T) \mathrm{t} \mathrm{t}\left[\mathbb{1}-r(\omega) l^{\dagger}(\omega)-t(\omega) t^{\dagger}(\omega)\right],
\end{gathered}
$$




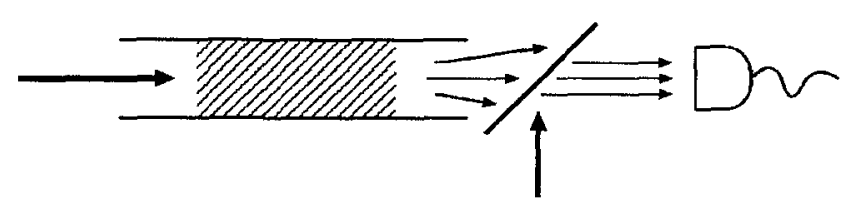

FIG 2 Schematic illustration of homodyne detection At the left, radiation is incident on a random medium (shaded) At the right, a strong coherent beam is superimposed onto the transmitted radiation, and the combined radiation is absorbed by a photodetector

$$
\begin{aligned}
& \kappa_{2}=2 I_{0} \tau d^{2} f\left(\omega_{0}, T\right)\left[t^{\dagger}\left(\mathbb{1}-r^{\dagger}-t t^{\dagger}\right) t\right]_{m_{0} m_{0}}+\kappa_{2}^{\mathrm{th}}, \quad \\
& \kappa_{2}^{\mathrm{th}}=\tau d^{2} \int_{0}^{\sigma} \frac{d \omega}{2 \pi} f^{2}(\omega, T) \mathrm{t}\left[1-1(\omega) r^{\dagger}(\omega)-t(\omega) t^{\dagger}(\omega)\right]^{2}
\end{aligned}
$$

Here $I_{0}=(2 \pi)^{-1} \int_{0}^{\infty} d \omega|\alpha|^{2}$ is the incident photon flux and the matices $r$ and $t$ without frequency algument ale to be evaluated at frequency $\omega_{0}$ These are the results of Ref [11]

\section{HOMODYNE DETECTION}

The photocount measurement described in Sec III (known as direct detection) cannot distinguish between the two quadratures of the electric field Such phase-dependent information can be retrieved by homodyne detection, $1 \mathrm{e}$, by superimposing a strong piobe beam (described by operators $a^{\text {probe }}$ ) onto the signal beam (see $F_{1} g$ 2) The total radiation incident on the detector is described by the operator

$$
a^{\text {total }}=\kappa^{1 / 2} a^{\text {out }}+(1-\kappa)^{1 / 2} a^{\text {probe }}
$$

where the factor $\sqrt{\kappa}$ accounts for the attenuation of the signal beam by the beam splitter that superimposes it onto the probe beam (For simplicity we assume a real scalar $\kappa$, more generally $\kappa$ would be a complex coupling matrix )

The characteristic function of $a^{\text {total }}$ is the product of the characteristic functions of $a^{\text {out }}$ and $a^{\text {probe }}$ We assume that the probe beam is in the coheient state with displacement vector $\beta$, having elements $\beta_{n p}=\beta_{n}\left(\omega_{p}\right)$ Fiom Eq (211) one gets

$$
\begin{aligned}
\chi_{\text {total }}(\eta)= & \exp \left[-\kappa \eta^{\dagger}\left(\mathbb{1}-S S^{\dagger}\right) f \eta+(1-\kappa)^{1 / 2}\left(\beta^{\dagger} \eta-\eta^{\dagger} \beta\right)\right] \\
& \times \chi_{1 \mathrm{n}}\left(S^{\dagger} \kappa^{1 / 2} \eta\right)
\end{aligned}
$$

The generating function $F_{\text {homo }}(\xi)$ of the photocount distribution in homodyne detection is given by [cf $\mathrm{Eq}(36)]$

$$
\begin{aligned}
\exp \left[F_{\text {homo }}(\xi)\right]= & \left\langle\exp \left(\xi \Delta a^{\text {total }} \mathcal{D} a^{\text {total }}\right)\right\rangle \\
\approx & \exp \left[\xi(1-\kappa) \beta^{\dagger} \mathcal{D} \beta\right]\left\langle\operatorname { e x p } \left(\Delta^{1 / 2} \sqrt{\kappa(1-\kappa)} \xi\right.\right. \\
& \left.\left.\times\left[a^{\text {out } \dagger} \mathcal{D} \beta+\beta^{\dagger} \mathcal{D} a^{\text {out }}\right]\right)\right\rangle
\end{aligned}
$$

In the second approximate equality we have linearized the exponent with respect to $a^{\text {out }}$, which is justified if the probe beam is much stronger than the signal beam The remaining expectation value has the form of a characteristic function if we take $\xi$ purely imaginaly, so that $\xi^{*}=-\xi$ The result is

$$
\begin{aligned}
F_{\text {homo }}(\xi)= & \xi(1-\kappa) \beta^{\dagger} \mathcal{D} \beta+\ln \chi_{\text {out }}(\sqrt{\kappa(1-\kappa)} \xi \mathcal{D} \beta) \\
= & \xi(1-\kappa) \beta^{\dagger} \mathcal{D} \beta+\kappa(1-\kappa) \xi^{2} \beta^{\dagger} \mathcal{D}\left(\mathbb{1}-S S^{\dagger}\right) f \mathcal{D} \beta \\
& +\ln \chi_{\mathrm{In}}\left(\sqrt{\kappa(1-\kappa)} \xi S^{\dagger} \mathcal{D} \beta\right)
\end{aligned}
$$

In the second equation we have substituted the relation (2 11) between $\chi_{\text {out }}$ and $\chi_{\mathrm{in}}$

\section{SQUEEZED RADIATION}

We consider the case that the incident radiation is in the ideal squeezed state $|\epsilon, \alpha\rangle=\mathcal{C S}|0\rangle[1,2]$, obtaned fiom the vacuum state $|0\rangle$ by subsequent action of the squeezing ope1ator

$$
\mathcal{S}=\exp \left[\frac{1}{2} \Delta\left(a^{\mathrm{ln}} \epsilon^{*} a^{\mathrm{In}}-a^{\mathrm{ln} \dagger} \epsilon a^{\mathrm{ln} \dagger}\right)\right]
$$

and the displacement operator

$$
\mathcal{C}=\exp \left[\Delta^{1 / 2}\left(a^{1 \mathrm{n} \dagger} \alpha-\alpha^{\dagger} a^{\mathrm{mn}}\right)\right]
$$

As in the preceding sections, we have discretized the fiequency, $\omega_{p}=p \Delta$, and used the vector of operators $a_{n p}^{\text {in }}$ $=a_{n}^{\text {nn }}\left(\omega_{p}\right)$ The complex squeezing parameters $\epsilon_{n}(\omega)$ $=\rho_{n}(\omega) e^{l \phi_{n}(\omega)}$ are contaned in the diagonal matrix $\epsilon$ with elements $\epsilon_{n p n^{\prime} p^{\prime}}=\epsilon_{n}\left(\omega_{p}\right) \delta_{n n^{\prime}} \delta_{p p^{\prime}}$ Similarly, the vector $\alpha$ with elements $\alpha_{n p}=\alpha_{n}\left(\omega_{p}\right)$ contains the displacement parameters

The characteistic function of the incident radiation is given by $[1,19]$

$$
\begin{aligned}
\chi_{\mathrm{nn}}(\eta)= & \exp \left[\alpha^{\dagger} \eta-\eta^{\dagger} \alpha-\frac{1}{4} \eta^{T}\left(e^{-i \phi} \sinh 2 \rho\right) \eta\right. \\
& \left.-\frac{1}{4} \eta^{\dagger}\left(e^{l \phi} \sinh 2 \rho\right) \eta^{\sharp}-\eta^{\dagger}\left(\sinh ^{2} \rho\right) \eta\right]
\end{aligned}
$$

According to Eq (211), we thus find for the characteistic function of the outgoing radiation

$$
\begin{aligned}
\chi_{\text {out }}(\eta)= & \exp \left(\alpha^{\dagger} S^{\dagger} \eta-\eta^{\dagger} S \alpha-\frac{1}{4} \eta^{T} S^{\dagger}\left(e^{-\imath \phi} \sinh 2 \rho\right) S^{\dagger} \eta\right. \\
& -\frac{1}{4} \eta^{\dagger} S\left(e^{\imath \phi} \sinh 2 \rho\right) S^{T} \eta^{*} \\
& \left.-\eta^{\dagger}\left[f-S\left(f-\sinh ^{2} \rho\right) S^{\dagger}\right] \eta\right)
\end{aligned}
$$

The generating function $F(\xi)$ of the photocount disti1bution is obtained fiom $\chi_{\text {in }}$ by convolution with a Gaussian, cf Eq (37) We find

$$
F(\xi)=F_{\mathrm{th}}(\xi)-\frac{1}{2} \ln \operatorname{det} X-\frac{1}{2}\left(\begin{array}{c}
\alpha^{*} \\
\alpha
\end{array}\right)^{T} X^{-1}\left(\begin{array}{c}
M \alpha \\
M^{*} \alpha^{*}
\end{array}\right),
$$

where the matrix $X$ is defined in terms of the matrix $M$ by

$$
\begin{aligned}
X= & I+\left(\begin{array}{cc}
M \sinh \rho & -M e^{\imath \phi} \cosh \rho \\
-M^{*} e^{-\imath \phi} \cosh \rho & M^{*} \sinh \rho
\end{array}\right) \\
& \times\left(\begin{array}{cc}
\sinh \rho & 0 \\
0 & \sinh \rho
\end{array}\right)
\end{aligned}
$$

If squeezing is absent, $\rho=0$, hence $X=\mathbb{1}$ and Eq (5 5) reduces to the result $(316)$ for coherent 1adiation For a squeezed vacuum $(\alpha=0)$ one has simply $F(\xi)=F_{\mathrm{th}}(\xi)$ $-\frac{1}{2} \ln \operatorname{det} X$ 
If the radiation is incident only in mode $m_{0}$, then we may compute the matrix inverse and the determinant in Eq (5 5) explicitly The matix $M(\omega)$ defined in Eq (311) may be replaced by its $m_{0}, m_{0}$ element,

$M_{m_{0} m_{0}}(\omega) \equiv m=-\xi\left(S^{\dagger}\left(1-\xi D\left[1-S S^{\dagger}\right] f\right)^{-1} D S\right)_{m_{0} m_{0}}$

Note that $m$ is real, since it is the diagonal element of a Hetmitian matix The resulting generating function is

$$
\begin{aligned}
F(\xi)= & F_{\mathrm{th}}(\xi)-\frac{1}{2} \tau \int_{0}^{\infty} \frac{d \omega}{2 \pi} \ln \left(1+2 m \sinh ^{2} \rho-m^{2} \sinh ^{2} \rho\right) \\
& -\tau \int_{0}^{\infty} \frac{d \omega}{2 \pi} m|\alpha|^{2} \\
& \times \frac{1+m \sinh \rho[\sinh \rho+\cosh \rho \cos (2 \arg \alpha-\phi)]}{1+2 m \sinh ^{2} \rho-m^{2} \sinh ^{2} \rho}
\end{aligned}
$$

The first two factorial cumulants, for detection in tiansmission, are

$$
\begin{aligned}
\kappa_{1}=\kappa_{1}^{\mathrm{th}}+\tau d \int_{0}^{\infty} \frac{d \omega}{2 \pi}\left(|\alpha|^{2}+\sinh ^{2} \rho\right)\left[t^{\dagger} t\right]_{m_{0} m_{0}} \\
\kappa_{2}=\kappa_{2}^{\mathrm{th}}+2 \tau d^{2} \int_{0}^{\infty} \frac{d \omega}{2 \pi}\left(|\alpha|^{2}+\sinh ^{2} \rho\right) \\
\quad \times f\left[t^{\dagger}\left(1-r r^{\dagger}-t t^{\dagger}\right) t\right]_{m_{0} m_{0}}+\tau d^{2} \int_{0}^{\infty} \frac{d \omega}{2 \pi}\left[t^{\dagger} t\right]_{m_{0} m_{0}}^{2} \\
\quad \times\left[\mid \alpha \cosh \rho-\alpha^{\dagger} e^{\imath \phi}{\left.\sinh \rho\right|^{2}-|\alpha|^{2}}+\sinh ^{2} \rho\left(\cosh ^{2} \rho+\sinh ^{2} \rho\right)\right]
\end{aligned}
$$

where $\kappa_{1}^{\text {th }}$ and $\kappa_{2}^{\text {th }}$ are given by Eqs (3 21) and (3 23)

The generating function for homodyne detection follows from Eqs (4 5) and (5 4),

$$
\begin{aligned}
F_{\text {homo }}(\xi)= & \xi(1-\kappa) \beta^{\dagger} \mathcal{D} \beta+\xi \sqrt{\kappa(1-\kappa)} \\
& \times\left(\alpha^{\dagger} S^{\dagger} \mathcal{D} \beta+\beta^{\dagger} \mathcal{D} S \alpha\right)-\frac{1}{4} \xi^{2} \kappa(1-\kappa) \\
& \times\left[\beta \mathcal{D} S^{\star}\left(e^{-\imath \phi} \sinh 2 \rho\right) S^{\dagger} \mathcal{D} \beta\right. \\
& \left.+\beta^{\dagger} \mathcal{D} S\left(e^{\imath \phi} \sinh 2 \rho\right) S^{T} \mathcal{D} \beta^{\star}\right] \\
& +\xi^{2} \kappa(1-\kappa) \beta^{\dagger} \mathcal{D}\left[f-S\left(f-\sinh ^{2} \rho\right) S^{\dagger}\right] \mathcal{D} \beta
\end{aligned}
$$

All factorial cumulants except for the first two vanish in the strong-probe approximation We may simplify the generating function by assuming that the signal beam is incident in a single mode $m_{0}$ and that the probe beam is also in a single mode $n_{0}$ For detection in transmission one then has the factorial cumulants

$$
\kappa_{1}=\tau d \int_{0}^{\infty} \frac{d \omega}{2 \pi}\left\{(1-\kappa)|\beta|^{2}+2 \sqrt{\kappa(1-\kappa)} \operatorname{Re}\left[\alpha \beta^{*} t_{n_{0} m_{0}}\right]\right\}
$$

$$
\begin{aligned}
\kappa_{2}= & -\tau \kappa(1-\kappa) d^{2} \int_{0}^{\infty} \frac{d \omega}{2 \pi} \operatorname{Re}\left[\beta^{* 2} e^{1 \phi} t_{n_{0} m_{0}}^{2}\right] \sinh 2 \rho \\
& +2 \tau \kappa(1-\kappa) d^{2} \int_{0}^{\infty} \frac{d \omega}{2 \pi}|\beta|^{2}\left[\left|t_{n_{0} m_{0}}\right|^{2} \sinh ^{2} \rho\right. \\
& \left.+f\left(1-r r^{\top}-t t^{\dagger}\right)_{n_{0} n_{0}}\right]
\end{aligned}
$$

\section{FANO FACTOR}

For the application of these general formulas we focus oui attention on the Fano factor $\mathcal{F}$, defined as the ratio of the nowse power $P=\tau^{-1}$ vat $n$ and the mean curient $\bar{I}$ $=\tau^{-1} \bar{n}$

$$
\mathcal{F}=P / \bar{I}=1+\kappa_{2} / \kappa_{1}
$$

(We have assumed the limit $\tau \rightarrow \infty$ ) For coheient radiation $\mathcal{F}=1$, corresponding to Poisson statistics Theimal iadiation has $\mathcal{F}>1$ (super-Poissonian) Nonclassical states, such as squeezed states, can have $\mathcal{F}<1$

We assume that the radiation is incident in a single mode $m_{0}$ and 1 s detected in transmission (equal efficiency $d$ per transmitted mode) We consider a frequency-iesolved measuiement, covering a nariow fiequency interval around the central frequency $\omega_{0}$ of the incident radiation The the1mal contributions $\kappa_{1}^{\text {th }}$ and $\kappa_{2}^{\text {th }}$ may then be neglected, since they are spread out ovel a wide frequency range The incident radiation has Fano factor $\mathcal{F}_{\text {in }}$, measured in direct detection with unit efficiency For squeezed radiation, one has

$$
\mathcal{F}_{\mathrm{In}}=1+\frac{\left|\alpha \cosh \rho-\alpha^{*} e^{\iota \phi} \sinh \rho\right|^{2}-|\alpha|^{2}+\sinh ^{2} \rho\left(\cosh ^{2} \rho+\sinh ^{2} \rho\right)}{|\alpha|^{2}+\sinh ^{2} \rho}
$$


We seek the Fano factor of the transmitted radiation, both for direct detection $\left(\mathcal{F}_{\text {drect }}\right)$ and for homodyne detection ( $\mathcal{F}_{\text {homo }}$ ) Combinıng Eqs (5 9) and (5 10), we find for direct detection

$$
\begin{aligned}
\mathcal{F}_{\text {drect }}-1= & d\left(t^{\dagger} t\right)_{m_{0} m_{0}}\left(\mathcal{F}_{1 \mathrm{n}}-1\right) \\
& +2 d f\left(\omega_{0}, T\right) \frac{\left[t^{\dagger}\left(1-r r^{\dagger}-t t^{\dagger}\right) t\right]_{m_{0} m_{0}}}{\left(t^{\perp} t\right)_{m_{0} m_{0}}}
\end{aligned}
$$

The first term is due entirely to the incident radiation It is absent for coherent radiation (because then $\mathcal{F}_{\text {m }}=0$ ) The second term is due to the beating of the incident radiation with the vacuum fluctuations It is independent of the incident radiation and was studied in detail in Ref [11] Sub Poissonian counting statıstics, $1 \mathrm{e}, \mathcal{F}_{\text {direct }}<1$, is in an ampltfying medium $(f \leqslant-1)$ only possible when

$$
2\left(t^{\dagger} t t^{\dagger} t\right)_{m_{0} m_{0}}<\left(t^{\dagger} t\right)_{m_{0} m_{0}}^{2}+2\left(t^{\dagger} t\right)_{m_{0} m_{0}}-2\left(t^{\dagger} r r^{\dagger} t\right)_{m_{0} m_{0}}
$$

In the absence of reflection $(r=0)$ and intermode scatteing ( $t$ diagonal), this reduces to the well-known condition [20-22] $\left(t^{\dagger} t\right)_{m_{0} m_{0}}<2$ Since $\left(t^{\dagger} t t^{\dagger} t\right)_{m_{0} m_{0}}=\Sigma_{k}\left|\left(t^{\dagger} t\right)_{m_{0} k}\right|^{2}$ $\geqslant\left(t^{\dagger} t\right)_{m_{0} m_{0}}^{2}$, the presence of intermode scattering decreases the maximally allowed amplification factor $\left(t^{\perp} t\right)_{m_{0} m_{0}}$

The Fano factor in the strong-probe approximation $(|\beta| \rightarrow \infty)$ follows f1om Eqs (5 12) and (5 13), w1th the result

$$
\begin{aligned}
\mathcal{F}_{\text {homo }}-1= & 2 d \kappa\left|t_{n_{0} m_{0}}\right|^{2} \sinh ^{2} \rho+2 d \kappa f\left(\omega_{0}, T\right) \\
& \times\left(1-r r^{\dagger}-t t^{\dagger}\right)_{n_{0} n_{0}}-d \kappa \\
& \times \operatorname{Re}\left[e^{l(\phi-2 \mathrm{dg} \beta)} t_{n_{0} m_{0}}^{2}\right] \sinh 2 \rho
\end{aligned}
$$

In the strong-probe approxımation, it is independent of $\alpha$ and $|\beta|$ Similarly to $\mathrm{Eq}$ (63), the first term is entirely due to the incident radiation, vanishing for coherent radiation $(\rho=0)$, and the second term is due the beating with vacuum fluctua tions The additional third term describes the effect of the phase of the probe beam on the measurement Typically, in a measurement one would vary the phase of the probe beam until the Fano factor is minımized, which occurs when $\arg \beta=\frac{1}{2} \phi+\arg t_{n_{0} m_{0}}$ The resulting Fano factor $\mathcal{F}_{\text {homo }}^{\min }$ is given by
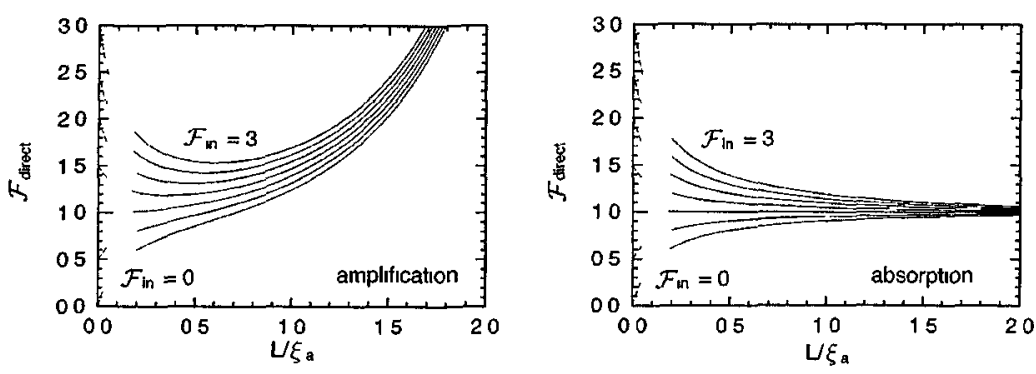

$$
\begin{aligned}
\mathcal{F}_{\text {homo }}^{\min }= & 1-2 d \kappa\left|t_{n_{0} m_{0}}\right|^{2} e^{-\rho} \sinh \rho \\
& +2 d \kappa f\left(\omega_{0}, T\right)\left(1-\imath r^{\dagger}-t t^{\dagger}\right)_{n_{0} n_{0}}
\end{aligned}
$$

Squeezing in the outgoing radiation for an amplifying medium is only possible when $\left|t_{n_{0} m_{0}}\right|^{2}<2-2\left(r^{\dagger}\right)_{n_{0} n_{0}}$ $-\Sigma_{k \neq m_{0}}\left|t_{n_{0} k}\right|^{2}$ The single-mode limit $\left|t_{n_{0} m_{0}}\right|^{2}=2$ is thus decreased by both reflection and intermode scattering

\section{ENSEMBLE AVERAGES}

The expressions for the Fano factor given in the preceding section contain the reflection and tiansmission matices of the waveguide These ate $N$ dimensional matrices that de pend on the positions of the scatteiers inside the waveguide The distiıbution of these matices in an ensemble of disordered waveguides is described by random matix theory [12] Ensemble averages of moments of $r t^{2}$ and $t t^{\dagger}$ for $N$ $\gg 1$ have been computed by Brouwer [23], as a function of the mean free path $l$ and the amplification (absorption) length $\xi_{a}=\sqrt{D \tau_{a}}$, where $1 / \tau_{a}$ is the amplification (absorption) rate and $D=c l / 3$ is the diffusion constant It is assumed that both $\xi_{a}$ and $L$ are small compared to the localization length $N l$ but large compared to the mean free path $l$ Obviously, this tequires a large number $N$ of propagating modes The relative size of $L$ and $\xi_{a}$ is arbitiary

As sample-to sample fluctuations are small for $N \gg 1$, we can take in Eq (6 3) the averages of numerator and denomnator separately The dependence on the index $m_{0}$ of the incident mode diops out on averaging, \langle\rangle$_{m_{0} m_{0}}$ $=N^{-1}\langle\operatorname{tr}\rangle$ For an absorbing disoldeied waveguide, we find

$$
\begin{aligned}
\mathcal{F}_{\text {drect }}= & 1+\frac{4 l d}{3 \xi_{a} \sinh s}\left(\mathcal{F}_{\text {In }}-1\right)+\frac{d}{2} f\left(\omega_{0}, T\right) \\
& \times\left[3-\frac{2 s+\operatorname{cotanh} s}{\sinh s}-\frac{s \operatorname{cotanh} s-1}{\sinh ^{2} s}+\frac{s}{\sinh ^{3} s}\right]
\end{aligned}
$$

We have abbreviated $s=L / \xi_{a}$ In the limit of stiong absoip tion, $s \rightarrow \infty$, the Fano factor appioaches the universal limit [24] $\mathcal{F}_{\text {direct }}=1+\frac{3}{2} d f$ The Fano factor $\mathcal{F}_{\text {in }}$ is given by Eq (62) for an incident squeezed state, but $\mathrm{Eq}$ (7 1) is more generally valid for any state of the incident radiation

The result for an amplifying disordered waveguide follows by the replacement $\tau_{a} \rightarrow-\tau_{a}$, hence $\xi_{a} \rightarrow l \xi_{a}$
FIG 3 Average Fano factor $\mathcal{F}_{\text {ducct }}$ for direct detection as a function of the length of the wave guide The left panel is for an amplifying medium [Eq (7 2), $f=-1$ ], the right panel for an absorbing medium $[\mathrm{Eq}(71), f=0]$ In both cases we took $l / \xi_{a}=01, d=1$, and values of $\mathcal{F}_{\mathrm{ill}}$ increasing from 0 to 3 in steps of 05 The dotted parts of the curves are extrapolations in the range $L$ $\leq l$ that is not covered by Eqs (7 1) and (72) 

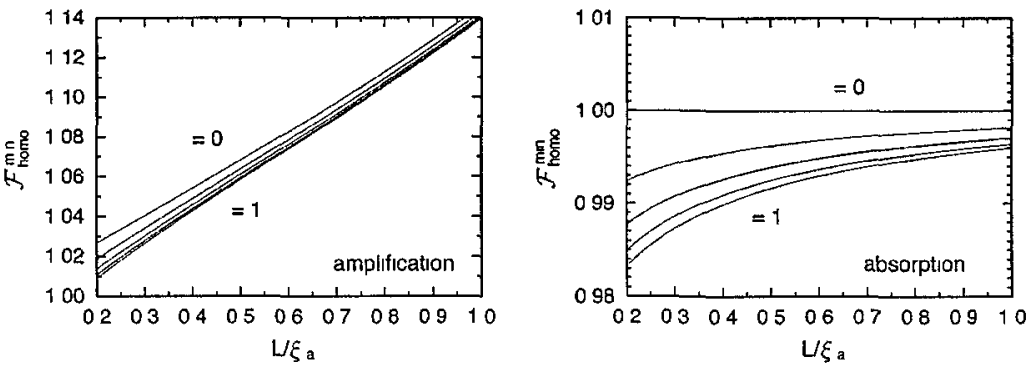

FIG 4 Average minimal Fano factor for homodyne detection, from Eqs (73) and (7 4) Same parameter values as in Fig 3 , with $N$ $=10, \kappa=\frac{1}{2}$, and $\rho$ increasing from 0 to 1 in steps of 025 For $L \leqslant l$ the curves extrapolate either to 1 (if $n_{0} \neq m_{0}$ ) or to $1-e^{-\rho} \sinh \rho$ (if $n_{0}=m_{0}$ ) (This extrapolation is not shown in the figure)

$$
\begin{aligned}
\mathcal{F}_{\text {direct }}= & 1+\frac{4 l d}{3 \xi_{a} \sin s}\left(\mathcal{F}_{\mathrm{ln}}-1\right)+\frac{d}{2} f\left(\omega_{0}, T\right) \\
& \times\left[3-\frac{2 s-\operatorname{cotan} s}{\sin s}+\frac{s \operatorname{cotan} s-1}{\sin ^{2} s}-\frac{s}{\sin ^{3} s}\right]
\end{aligned}
$$

The Fano factor diverges at the lase threshold $s=\pi$ The function $f\left(\omega_{0}, T\right)$ now has to be evaluated at a negative temperature For a complete population inversion of the atomic states $f \rightarrow-1$

The minimal Fano factor in homodyne detection is given by Eq (66) The average $\left\langle\left|t_{n_{0} m_{0}}\right|^{2}\right\rangle$ is again independent of the mode indices, hence it can be replaced by $N^{-2}\left\langle\operatorname{tr} t t^{\dagger}\right\rangle$ For an absorbing wavegurde we find

$$
\begin{aligned}
\mathcal{F}_{\text {homo }}^{\min }= & 1-\frac{8 l d \kappa}{3 N \xi_{a} \sinh s} e^{-\rho} \sinh \rho+\frac{8 l d \kappa}{3 \xi_{a}} f\left(\omega_{0}, T\right) \\
& \times\left[\operatorname{cotanh} s+\frac{1}{\sinh s}\right],
\end{aligned}
$$

and for an amplifying waveguide

$$
\begin{aligned}
\mathcal{F}_{\text {homo }}^{\min }= & 1-\frac{8 l d \kappa}{3 N \xi_{a} \sin s} e^{-\rho} \sinh \rho \\
& +\frac{8 l d \kappa}{3 \xi_{a}} f\left(\omega_{0}, T\right)\left[\operatorname{cotan} s-\frac{1}{\sin s}\right]
\end{aligned}
$$

Measuiement of the ensemble average $\mathcal{F}$ homo requiles that for every sample the phase of the probe beam is readjusted so as to minimize the Fano factor This is common practice in a homodyne measurement If the phase of the probe beam is fixed, the random phase of $t_{n_{0} m_{0}}$ w1ll average to zero the third term in Eq (6 5) In Eqs (7 3) and (7 4) this amounts to the substitution $e^{-\rho} \rightarrow-\sinh \rho$
A giaphical presentation of the results (7 1)-(7 4) is given in Figs 3-5 For the absorbing case we have taken $f=0$ (appiopilate for optical frequencies at $100 \mathrm{~m}$ temperature) For the amplifying case we have taken $f=-1$ (complete population inversion) The formulas above cannot be used for $L \leqq l$ The values of $\mathcal{F}_{\text {direct }}, \mathcal{F}_{\text {homo }}$, and $\mathcal{F}$ homo for $L=0$ can be read off from Eqs (63)-(66), $\mathcal{F}_{\text {drrect }}$ $\rightarrow 1+d\left(\mathcal{F}_{\text {in }}-1\right), \mathcal{F}_{\text {homo }}=1+2 \delta_{n_{0} m_{0}} d \kappa \sinh ^{2} \rho$, and $\mathcal{F}_{\text {homo }}^{\text {min }}$ $=1-2 \delta_{n_{0} m_{0}} d \kappa e^{-\rho} \sinh \rho$ An extrapolation to $L=0$ is shown by dashes in Fig 3

The common feature of the Fano factors plotted in Figs 3-5 is a convergence as the length of the waveguide becomes longer and longer For an absorbing medium the $L \rightarrow \infty$ limit is independent of the state of the incident radiation For an amplifying medium, complete convergence is preempted by the laser threshold at $L=\pi \xi_{a}$

\section{CONCLUSIONS}

In conclusion, we have derived general expiessions for the photodetection statistics in teims of the scatteing matrix of the medium through which the radiation has propagated These expressions are paiticularly well surted for evaluation by means of 1 andom-matrix theory, as we have shown by an explicit example, namely the propagation of squeezed radia tion through an amplifying or absorbing waveguide The sub Possonian noise that can occur in a squeezed state (chatacterized by a Fano factor smaller than unity) is destroyed by theimal fluctuations in an absorbing medium or by spontaneous emission in an amplifying medium The theory presented here describes this interaction of nonclassical radiation with matter in a quantitative way, without the restriction to one-dimensional scattermg of earlieı investigations

\section{ACKNOWLEDGMENTS}

This work was supported by the Nederlandse Oiganısatie voor Wetenschappelık Ondeızoek (NWO) and the Stıchtıng voor Fundamenteel Onderzoek der Mateı (FOM)
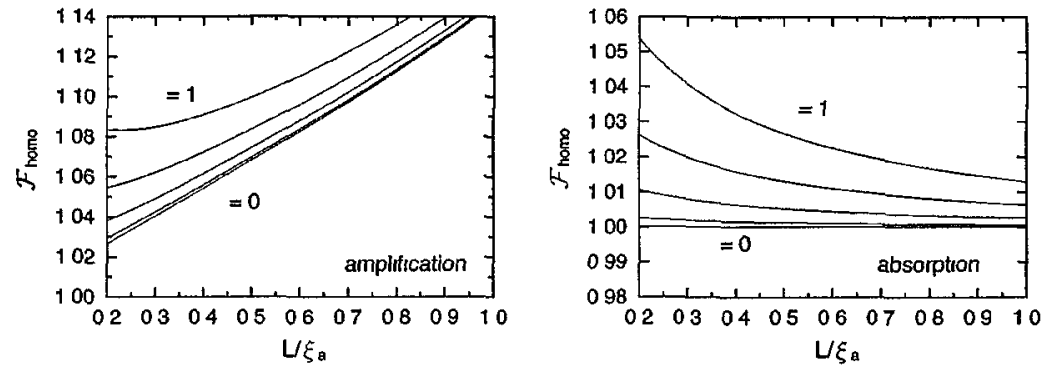

$063805-7$
FIG 5 Average Fano factor for homodyne detection, from Eqs (73) and (7 4) after the substitution $e^{-\rho} \rightarrow-\sinh \rho$, otherwise identical to Fig 4 For $L \leqq l$ the curves extrapolate either to 1 (if $n_{0} \neq m_{0}$ ) or to $1+\sinh ^{2} \rho$ (if $n_{0}=m_{0}$ ) (This extrapolation is not shown in the figure) 
[1] D F Walls and G J Milburn, Quantum Optics (Springer, Berlın, 1994)

[2] L Mandel and E Wolf, Optical Coherence and Quantum $O p$ tıcs (Cambridge University Press, New York, 1995)

[3] C H Henry and R F Kazannov, Rev Mod Phys 68, 801 (1996)

[4] U Leonhardt, J Mod Opt 40, 1123 (1993)

[5] J Jeffers and S M Barnett, J Mod Opt 41, 1121 (1994)

[6] E Schmidt, L Knoll, and D-G Welsch, Phys Rev A 54, 843 (1996)

[7] S M Barnett, J Jeffers, A Gatt1, and R Loudon, Phys Rev A 57, 2134 (1998)

[8] M Artoni and R Loudon, Phys Rev A 59, 2279 (1999)

[9] L Knoll, S Scheel, E Schmidt, D-G Welsch, and A V Chizhov, Phys Rev A 59, 4716 (1999)

[10] G M Abd Al-Kader, Eur Phys J B 8, 429 (1999)

[11] M Patra and C W J Beenakker, Phys Rev A 60, 4059 (1999)

[12] C W J Beenakker, Rev Mod Phys 69, 731 (1997)
[13] J Jeffers, N Imoto, and R Loudon, Phys Rev A 47, 3346 (1993)

[14] T Gruner and D-G Welsch, Phys Rev A 54, 1661 (1996)

[15] C W J Beenakker, Phys Rev Lett 81, 1829 (1998)

[16] R J Glauber, Phys Rev Lett 10, 84 (1963)

[17] P L Kelley and W H Kleıner, Phys Rev 136, A316 (1964)

[18] C W J Beenakker, in Diffuse Waves in Complex Media, Vol 531 of NATO Advanced Study Instutute Series $C$, edited by J -P Fouque (Kluwer, Dordrecht, 1999)

[19] H P Yuen, Phys Rev A 13, 2226 (1976)

[20] C M Caves, Phys Rev D 26, 1817 (1982)

[21] R Loudon and T J Shepherd, Opt Acta 31, 1243 (1984)

[22] S Stenholm, Phys Scr T12, 56 (1986)

[23] P W Brouwer, Phys Rev B 57, 10526 (1998)

[24] C W J Beenakker and M Patıa, Mod Phys Lett B 13, 337 (1999), C W J Beenakkel, M Patra, and P W Brouwer, Phys Rev A 61, 051801(R) (2000) 